\title{
Neurofeedback in the enhancement of cognitive coherence in schizophrenic patients
}

\author{
Leibetseder M*, Pazooki KH, Renner W and Gruber D \\ Psychotherapy Science, Sigmund Freud University, Vienna, Austria
}

\begin{abstract}
In this study we discuss two single cases. These single-case studies referring to neurofeedback therapy with two patients displaying the negative symptoms of schizophrenia. Both patients struggle with developing coherent connections. Firstly, we would like to analyse the characteristics of progress in the therapeutic process, and secondly, evaluate its effectiveness. Following EEG parameters were measured: Mean-Theta, mean-Alpha, SMR, mean-Beta and mean-EMG. The coherence was measured by special the analysis of language protocols. The approach is deferred from Omanson's concepts [1]. The language was adapted and encompasses the linguistic characteristics of "central, "supportive" and "distractive". "Central" refers to any proposition containing an intentional act of the main actor, an intended disruption of their action or, a self-initiated enabling of the action. "Supportive" encompasses any proposition containing cognitions and feelings of the main character as well as important situations. Important situations are situations representing circumstances for the intended behaviour of the main character. "Distractive" are propositions without a main character as well as propositions containing people and situations that have no influence on the intended behaviour of the main character or those, which support the indirect "distractive". Propositions containing cognitions and emotions about "distractive" are also considered distractive in themselves. The hypotheses of the study are as follows: (1) Hypothesis concerning the evaluation of effect: There is a significant positive correlation between the measurement times and the language characteristics "central" and "supportive" and a negative correlation between measurement times and the "distractive" characteristic. (2) Hypothesis in regard to the process: There are significant correlations between the characteristics "central" and" supportive" and the EEG-variables mean-Alpha, SMR and negative correlations between the characteristics "central" and "supportive", mean-Theta and mean-Beta. ${ }^{2}$

${ }^{a *}$ Some parts of this paper have been taken form Khashyar Pazookìs Master Thesis at Sigmund Freud University, Vienna [2].

+ died 2018

(1) Sigmund Freud University Vienna, Vienna Austria

(2) Group Psylux, Neuroacademy \& Traumainstitut Luxembourg Cabinet de Psychologie \& de Sciences de la Psychothèrapie Khashayar Pazooki, Luxembourg, 53, route d`Arlon, 8211 Mamer, Luxembourg

(3) Pan - European University, Bratislava, Slovakia
\end{abstract}

\section{Introduction}

Characteristics of the negative symptoms of schizophrenia are affective flattening, social dysfunction, disturbance of abstract thinking, tangentiality, circumstantial thinking, incoherent and derailed thinking, uncooperative attitude, hostility, anxiety and guilt [3]. According to Andreasen, Andreasen, et al. [4] the cognitive characteristic of negative symptoms is "associative loosening". This looseness of associations is a differential characteristic of schizophrenic thought disorder. Hoffmann, et al. [5] use similar arguments in their research. The speech patterns of people diagnosed with schizophrenia as well as of those being manic is characterized by incoherence. People diagnosed with mania, combine one episode with another one, diverging thematically from the first one. The episodes within themselves are coherent. People with a schizophrenic illness display incoherence in the succession of episodes as well as within their inner structure.

The characteristics of the negative symptoms could be based on a disturbance of attention. Attention operates in a short-term storage of $100-500 \mathrm{~ms}$. The identification of the stimulus happens during this interval. Recognition of stimuli is (1) the extraction of a pattern from sensory characteristics and (2) the classification of his information in a conceptual category. Attention is fundamentally inhibitory. Irrelevant characteristics are inhibited. The inhibition of irrelevant stimuli enhances the effectiveness of the relevant ones [6].
The inhibitory mechanism of attentiveness shows for example in the alpha rhythm in an EEG. In people displaying the negative symptoms of schizophrenia, this inhibitory mechanism should be less pronounced. That means: The lesser the inhibition, the higher the contamination. Increased Theta activity has been correlated with deficits in attentiveness [7]. This pattern indicates a state of decreased alertness, respectively an increased disposition for sleep and relaxation. Increased Beta activity suggests that attention deficits are based on a hyperactivity [8].

During neurofeedback training, people get a return of these chronological patterns via a screen. This feedback should allow the concerned subjects to regulate the inhibitory processes. As such, overexcitement, inattention, under-excitement, instability or disinhibition should be identified and changed. A stabilization of SMR (between 12 and $15 \mathrm{~Hz}$ ) is strived for. This pattern coincides with a

${ }^{\star}$ Correspondence to: Leibetseder M, Buchholzhofst.5, 5020, Salzburg, Austria, Tel: +43 67761021, E-mail: max.leibetseder@inode.at

Received: March 06, 2019; Accepted: March 12, 2019; Published: March 15, 2019 
relaxed state of the sensorimotor alertness. The coherence of speech protocols should increase.

The present study is a case study. As such, Döring, et al. [9] identify a possibility to comprehensively analyse a typical or untypical individual case. In regard to quantitative "...case studies with quasiexperimental designs...” repeated measurements are realized on the subject ... "This concept" aims to double-check the impact of different conditions. The present study analyses the course of a therapeutic intervention.

\section{It analyses:}

1. whether, during a neuro-feedback session with subjects displaying negative symptoms, there is a change in the pattern of -therapy variables of the EEG-

2. whether the coherence of thinking and speaking of subjects displaying negative symptoms can be changed during neurofeedback interventions

3. possible interactions of variables of the EEG pattern and characteristics of speech.

The analysis of speech protocols orients itself on a quantitative content analysis. According to Döring, et al. [9] a quantitative content analysis is based on a deductive derivate fully standardized system of categories, whose quality can be argued. Omanson system is a concept analyzing language [10]. It captures the coherence of texts. As such it differentiates between central and significant sentences and irrelevant sentences respectively distracters. The present study relates to Omanson's system. The content categories of this approach were defined by determinable, linguistic criteria.

\section{Procedure}

\section{Therapy}

This study analyses the process of the therapeutic intervention through neurofeedback as well as the effectiveness of the therapeutic process. The study had two participants (K.T. und B. Z.). They suffered from negative symptoms of schizophrenia.

The therapeutic intervention encompassed 20 individual sessions. In the first 10 sessions the subjects received clear instructions in regard to the stages in therapy. In the next 10 sessions no instructions were given. The instructions given in the first 10 sessions were intended to be continued and induced by the concerned subjects themselves.

"We used Mind-Media Nexus-10 (MK-II) devices for neurofeedback. The protocol was planned for two weeks of SMR (12 $-15 \mathrm{~Hz}$ ) augmentation at the contra-lateral side to the handedness of the participants, i.e., C4 for the right handed female and $\mathrm{C} 3$ for the left-handed male participant. Four to eight $\mathrm{Hz}$ theta was inhibited at the same electrode site. During the second phase, we again augmented $12-15 \mathrm{~Hz}$ SMR at the contra-lateral side to the handedness of both participants and inhibited four to eight $\mathrm{Hz}$ theta at the same electrode site. Additionally, we augmented beta - I $(13-18 \mathrm{~Hz})$ at F3 electrode site for both patients and four to eight $\mathrm{Hz}$ theta was inhibited at this electrode site" [2].

\section{Questions}

(a) Do the characteristics of negative symptoms change before and after neurofeedback?
(b)Is there a difference between the 10 sessions where explicit, clear instructions were given and the 10 sessions where no instructions were given?

(c) Are there correlations between EEG variables and language characteristics?

(d)Are there correlations between the preceding and the follow-up sessions and between EEG variables and language characteristics?

(e) Are there correlations between the EEG parameters and coherence characteristics and the language samples taken over the sessions?

\section{Variables}

a. Independent variable:

Sessions $1-20=$ time

b. Dependent variable $=$ Process variable $\mathrm{EEG}-$ parameter:

Mean Theta: The amplitude average of theta in $\mu \mathrm{V} 233$

Mean Alpha: The amplitude average of alpha in $\mu \mathrm{V} 234$

Mean SMR: The amplitude average of SMR in $\mu \mathrm{V} 235$

Mean Beta: The amplitude average of beta in $\mu \mathrm{V} 236$,

Mean EMG: The amplitude average of the artefacts in $\mu \mathrm{V}$

c. Dependent variable $=$ scales to examine the effectiveness of therapy:

$G A F$ - score was captured before and after therapy sessions and shows the changes in symptoms. The GAF - Scale (Global Assessment of Functioning Scale) is an instrument that evaluates overall functioning [13]. The objectivity of this scale is considered "relatively high ". Interrater reliability is - depending on the study - ICC $=0.81$; ICC $=0.89$ to ICC $=0.95[10-12]$. Reliability is variable - according to Lange, et al. [14] varying depending on the study between $r=0.54$ and $r=0.95$. Woldoff, Deschner reports values from $r=0.98$ to $r=$ $0.87[15,16]$. This yield- taking into account Fisher's $Z$ - an average reliability coefficient of about $\mathrm{r}=0.925$ to $\mathrm{r}=0.94$.

CompACT-SR captures alertness and selective attention [17]. Subjects are asked to respond to stimuli combined with distracting stimuli with a go or no-go answer. The reaction time is measured.

Coherence values of language samples after each session during the therapeutic process: variables of language protocols are as follows: central: supportive and distractive. They are formulated based on Omanson and language is adapted [1]. Wording of language categories and interpretation of protocols took place with and through a colleague and exact steps to be taken were agreed upon ahead of time. The categories are as follows:

Central are all propositions mentioning the main character and expressing intent and instrumental behavior of the main character. They can be identified by questions such as "why", and "how".

Supportive are all propositions containing thoughts and feelings of the main character as well as situations important for the main subject. Important situations represent conditions for intentional behavior of the main subject. They include a chronological sequence. This sequence reflects a conditional and consecutive "if - then" correlation. The consecutive element reflects a change in the probability of intended behavior of the main subject.

Distractive are all propositions describing secondary subjects as well as events without the main character, without an influence on "central, and supportive" - sentences. 
4. Statistical methods: all analyses will be carried out separately for each subject.

For question (a) the reliability change index will be computed.

Question (b) will be evaluated with a correlation analysis.

Question (c) will be evaluated with a cross correlation.

Question (d) will be evaluated with a canonical correlation.

\section{Results}

Question (a) relates to changes in overall functioning and selective attention. The general level of functioning was recorded with the GAF scale. The selective attention was measured with the CompACT-SR.

The changes in overall level of functioning and in selective attention were reevaluated with the Reliable Change Index (RCI). The RCI measures change in a measurement result that cannot be explained by an imminent measurement error of the instrument itself [18]. In this case a significance of $\alpha=0.05$ with the critical value of $\mathrm{z}$ crit. $=$ 1.65 has been suggested. The critical value for $\alpha=0.01$ is $\mathrm{z}$ crit. $=2.58$. According to Huber (1973) a significance level of $\alpha=0.10$ ( $\mathrm{z}$ crit. $=$ $1.28)$ is acceptable in individual case studies [19].

For subject T.K. the GAF values were: GAF $=51(\mathrm{z}=0.1)$ before and $\mathrm{GAF}=61(\mathrm{z}=1.1)$ after treatment. For subject B.Z. the values were GAF $=55(\mathrm{z}=0.5)$ before and GAF $=65(\mathrm{z}=1.5)$ after treatment. Assuming a reliability of $\mathrm{rtt}=0.87$, the $\mathrm{RCI}=5.56$. The GAF values before and after therapy significantly differ for both subjects.

For the subjects T.K.,and B.Z. the reaction times $(\mathrm{rtt}=0.95)$ before and after the treatment differed significantly $(\mathrm{RCI}=9.18$ and $\mathrm{RCT}=5.92)$. Both subjects displayed a statistically significant improvement in attention. At go-no go the selective attention for relevant stimuli under application of $0-4$ distractors is investigated. The RCIs for the subject T.K. are between RCI= 5.09 and 2.63. For the subject B.Z. they are between $\mathrm{RCI}=1.34$ and 0.09 . These achievements require an inner balance. With therapy, the selective attention of T.K. could be significantly improved in contrast to B.Z., who did not show significant improvement.

Question (b) was referring to the therapeutic process. This process was double-checked with a variance analysis [20]. Factor A concerns the therapeutic process across sessions. Factor B includes the condition "with" versus "without" clear instructions. Here, the validity of the null hypothesis is postulated. Both subjects should have implicitly continued the instructions formerly explained explicitly.

\section{- Participant B. Z.}

For the variable Mean $\theta$ : condition $A(\eta=0.15 ; z=3.26 ; p=0.01)$; condition $\mathrm{B}(\mathrm{F} 1 ; 5)=0.58$; n. s. $)$; interaction $\mathrm{AB}(\mathrm{F} 1 ; 9)=0.99$; n. s. $)$.

For Mean $\alpha$ : condition A $(\eta=0.32 ; z=2.96 ; p 0.01)$, condition B $(\mathrm{F} 1 ; 5) \mathrm{p}=0.53$; n. s.); interaction $\mathrm{AB}(\mathrm{F} 1 ; 9)=3.22 ; 0.05<\mathrm{p}<0.10)$.

For Mean SMR: condition A $(\eta=0.38 ; z=2.85 ; \mathrm{p}=0.01)$, condition $\mathrm{B}(\mathrm{F} 1 ; 5)=0.37$; n. s. $)$; interaction $\mathrm{AB}(\mathrm{F} 1 ; 9)=2.14$; n. s. $) .279$

For Mean $\beta$ : condition $A(\eta=0.25 ; z=3.06 ; p=0.01)$, condition $B$ $(\mathrm{F} 1 ; 5) \mathrm{p}=0.53$; n. s.), interaction $\mathrm{AB}(\mathrm{F} 1 ; 9) \mathrm{p}=0.58$; n. s.). 281

For Mean EMG: condition $\mathrm{A}(\eta=0.23 ; \mathrm{z}=3.13 ; \mathrm{p}=0.01)$, condition $\mathrm{B}(\mathrm{F} 1 ; 5)=1,26 ;$ n. s. $)$ interaction $\mathrm{AB}(\mathrm{F} 1 ; 9) \mathrm{p}=0.63$; n. s. $)$.
The critical values are as follows: $\mathrm{F}(1 ; 5) 5 \%=6,61$ and $\mathrm{F}(1 ; 5) 10 \%$ $=4,01 . \mathrm{F}(1 ; 9) 5 \%=5,12$ and $\mathrm{F}(1 ; 9) 10 \%=3.36[20]$.

\section{- Participant K. T.}

The results for variable Mean $\theta$ were: condition $A(\eta=0.02 ; z=$ $3.48 ; \mathrm{p}=0.01)$; condition $\mathrm{B}(\mathrm{F} 1 ; 5)=1.87$. n. s.) and for the interaction $\mathrm{AB}$ (F 1; 9), $\mathrm{p}=0.71$; n. s.).

For Mean $\alpha$ : condition $A(\eta=0.12 ; z=3.31 ; p=0.01)$, condition $B$ $(\mathrm{F} 1 ; 5)=1.52$; n. s.); interaction $\mathrm{AB}(\mathrm{F} 1 ; 9), \mathrm{p}=0.54$; n. s.).

For the variable Mean SMR results were: condition A $(\eta=0.08 ; \mathrm{z}$ $=3.17 ; \mathrm{p}=0.01), \mathrm{B}(\mathrm{F} 1 ; 5)=1.30 ; \mathrm{n} . \mathrm{s}$.$) , interaction \mathrm{AB}(\mathrm{F} 1 ; 9)=0.98$; n.s.).

For variable Mean $\beta$ the results were: condition $A(\eta=0.09 ; z=$ 3.34; $\mathrm{p}=0.01)$, condition $\mathrm{B}(\mathrm{F} 1 ; 5)=2.52 ; \mathrm{n} . \mathrm{s}$.$) ; interaction \mathrm{AB}(\mathrm{F} 1 ; 9)$ $\mathrm{p}=0.66$; n. s.).

For Mean EMG the results were: condition A ( $\eta=0.13 ; \mathrm{z}=3.29 ; \mathrm{p}$ $=0.01)$, for condition $\mathrm{B}(\mathrm{F} 1 ; 5)=0.95 ; \mathrm{n}$. s.); for the interaction $\mathrm{AB}(\mathrm{F}$ 1 ; 9) $\mathrm{p}=0.54$; n. s.).

It can be seen that for both participants and for all the EEG means, parameter differences across sessions were significant, i.e., in the course of neurofeedback, these means have changed. It is also evident that, for both participants and for all the variables assessed, there were no significant differences between the condition with instruction and the condition without instruction, i.e., the brain activities learned in the course of the training with instruction could be transferred successfully to situations were instructions were not present any longer.

Question (c) refers to the correlation between EEG parameters and language characteristics across all sessions. The simultaneous change of EEG and cognitive, linguistic variables during the therapeutic process is verified. The time interval between variables is here - to some extent - equal to zero.

- Subject B. Z. displays the following significant correlations:

Mean Alpha - central: $r=0.605(\mathrm{p}=0.005)$

Central - supportive: $r=-0.550(p=0.012)$

Supportive - distractive: $r=-0.642(p=0.002)$

Concerning subject B.Z., the language characteristics central and supportive and supportive and distractive correlate negatively. The more the focus of attention is directed toward key features, the less marginal and "solely" supporting features of an event are reported; and the more "at least" supportive aspects are called to mind, the less distracting and irritating, not fitting with the event, aspects have been reported. Central and the EEG variable mean alpha are positively correlated. Mean alpha promotes the selective, on central aspects focused attention. This result is consistent with the inhibition hypothesis of the alpha- variable (Klimesch, 2012) [6].

- Subject T.K. displays the following significant correlations:

Mean Theta - Mean Alpha: $r=0.986(p=0.000)$
Mean Theta - Mean SMR: $r=0.953(p=0.000)$
Mean Theta - Mean Beta: $r=0.867(p=0.000)$
Mean Theta - Mean EMG: $r=0.869(p=0.000)$
Mean Theta - central: $r=0.487(p=0.030)$
Mean Alpha - Mean SMR: $r=0.975(p=0.000)$ 
Mean Alpha - Mean Beta: $r=0.885(\mathrm{p}=0.000)$

Mean Alpha - Mean EMG: $r=0.886(\mathrm{p}=0.000)$

Mean SMR - Mean Beta: $\mathrm{r}=0.923(\mathrm{p}=0.000)$

Mean SMR - Mean EMG: $\mathrm{r}=0.953(\mathrm{r}=0.000)$

Mean Beta - Mean EMG: $r=0.949(r=0.000)$

Central - Supportive: $r=-757(p=000)$.

Characteristic for subject K.T. are the positive correlations between the EEG variables. Summarized these results mean: K.T. regulates the current state through inhibitory and antagonistic EEG characteristics. This creates an "inner balance".

Some examples of these interactions are:

- There is a significant correlation between Mean Theta and Mean Alpha; Mean Alpha has an inhibitory effect. The more inhibition, the "higher" the "reduced" wakefulness. Mean Alpha is significantly correlated with Mean Beta. The more inhibition on one hand, the more arousal on the other. This correlation refers to an antagonistic "compensation".

- There is a significant correlation between Mean Theta and Mean Beta. Mean Theta is an indicator of decreased alertness; Mean Beta is an indicator of increased alertness. The result means: the higher the probability of occurrence of Mean Beta, the higher the probability of occurrence of Mean Theta. Thus: "The higher the alertness", on one hand, the "smaller the alertness" on the other hand. Mean Theta does not inhibit mean-Beta, but is an antagonist to hyperarousal.

- The cognitive and linguistic characteristics of Central and supportive correlate negatively. This shows - even in the central of K.T., - that the focus of attention is geared toward key aspects of an event. Marginal and "solely" supporting features of an event are therefore rarely recalled. Mean Theta correlates positively with central. The therefore responsible neurophysiologic regulation mechanism is based on an increase of the EEG variable (Mean Theta), which reduces alertness [7].

The following correlations from B.Z. and K.T. are significantly different (whereby: d-crit. $(\alpha=0.01)$ : 0.92; (d-crit. $(\alpha=0.05)$ : 0.69)

- Mean Theta - Mean Alpha: dempirical. $=1.34$

- Mean Theta - Mean SMR: dempirical. $=1.84$

- Mean Theta - Mean Beta: dempirical. $=0.91$

- Mean Theta - Mean EMG: dempirical. $=1.11$

- Mean Alpha - Mean SMR: dempirical. $=2.13$

- Mean Alpha - Mean Beta: dempirical. = 1.01

- Mean Alpha - Mean EMG: dempirical. $=1.02$

- Mean SMR - Mean Beta: dempirical. $=1.52$

- Mean SMR - Mean EMG: dempirical. $=1.40$

- Mean Beta - Mean EMG: dempirical. $=1.32$

The correlations of subjects K.T. and B.Z. differ on all EEG variables; they do not differ on the linguistic characteristics. Thus, K.T. orients himself currently (in a prevailing way) as well as significantly, more on structural conditions of the EEG variables in comparison to B.Z. and Question (e) differentiate question (c). Question (c) is in regard to the correlations between the EEG - characteristics, language characteristics and the preceding and following therapeutic sessions. Here, the focus is on the influence of prior to follow-up sessions. Do variables, respectively changes in past changes, impact other variables at a later time of the therapeutic process? This question can be answered by using cross-correlations. Cross-correlations are correlations between changes of two characteristics that distinguish themselves through a defined time interval. Thus, the relationship of a characteristic at a given time with changes in other characteristics at prior or later times is examined. "Lag-" refers to a previous interval; "lag+" to a later interval. "Lag- «means that the variable $\mathrm{X}$ influences the variable Y, "subsequently, and in that regard later." Continues one the chronological course of comparisons, "Lag-" will become "Lag+". The chronological impact is thus reversed. "Lag+" means that variable Y will at a later time influence variable $\mathrm{X}$. Variable $\mathrm{X}$ is in turn influenced later by the variable Y. Question (e) analyses possible patterns between EEG- and linguistic variables.

\section{- The results for B.Z. are as follows:}

Mean Theta (lag -4) increases Mean Beta after four therapy sessions ( cross correlation coefficient $=.634$; standard error $=0.258$ )

Mean Theta (lag 2) is reduced from distractive after two sessions (cross correlation coefficient $=0 .-520$; standard error $=0.243$ )

Mean Alpha (lag -3) decreases after three therapy sessions Mean SMR (cross correlation coefficient $=0 .-634$; standard error $=0.250$ )

Mean Alpha decreases (lag -6) the language characteristic supportive after six therapy sessions (cross correlation coefficient $=0$.651 ; standard error $=0.277$ ).

Mean Alpha increases (lag -6) the language characteristic distractive after six therapy sessions. (cross-correlation coefficient $=$ 0.551 ; standard error $=0.277$ ).

Mean SMR decreases (lag-3) the language characteristic supportive after three therapy sessions (cross-correlation coefficient $=-0.581$; standard error $=0.250)$.

Mean SMR decreases (lag-3) the language characteristic distractive after three therapy sessions (cross-correlation coefficient $=-0.771$; standard error $=0.250$ ).

Mean Beta is reduced by the language characteristic central after three sessions (Lag3) (cross-correlation coefficient $=-0.771$; standard error $=0.250$ ).

Mean Beta increases the language characteristic central after three sessions. (lag-3) (cross-correlation coefficient $=0.534$; standard error $=0.250$ )

Mean Beta decreases the language characteristic central after 2 sessions. (lag -2) (cross-correlation coefficent $=-0.646$; Standard error $=0.243$ )

Mean Beta decreases the language characteristic supportive after three sessions (lag -3 ) (cross correlation coefficient $=-0.491$; standard error $=0.250$ ).

Mean Beta increases the language characteristic supportive after two sessions. (lag -2$)$ (cross correlation coefficient $=0.495$; standard error $=0.243$ )

Mean EMG decreases Mean SMR after three therapy sessions. (lag3) (cross-correlation coefficient $=-.571$; standard error $=0.250$ ). 
The results show correlations between EEG variables with cognitive, linguistic characteristics at a later point in time. The changes in the EEG - variables influence later changes in the cognitive processes and language characteristics and vice versa: cognitive processes in turn again later influence physiological processes.

\section{Examples:}

The cognitive irritability and distractibility through irrelevant details (distractive) decreases later on (more exactly: after two meetings) the decreased alertness (Mean Theta) (logically: “A double negative": the reduction of a reduction)

Hyperarousal (mean-Beta) furthers at a later point in time (more exactly: after three sessions) the focus on key characteristics (central), will however be decreased itself by this same characteristic.

Hyperarousal (mean-Beta) and the cognitive characteristics central and supportive "oscillate" at the same time. First, increased arousal increases (to be exactly: after three sessions) the focus of attention on key features and reduces the contribution to marginal, as only supportive, features. In a next step (to be exact: after two meetings), increased arousal reduces the focus for central characteristics and simultaneously increases the attention even to marginal details.

Mean SMR, referring to a relaxed sensorimotor alertness, will later on (to be exact: after three sessions) reduce the contribution to marginal and distractive details. This mechanism indirectly furthers coherence.

\section{Results for K.T. are as follows:}

The language characteristic central decreases after one session (lag $=-1)$ the language characteristic supportive (cross correlation coefficient $=-0.533$; standard error $=0.236$ )

The language characteristic central decreases after 4 sessions (lag $=-4$ ) distractive (cross- correlation coefficient $=-0.531$; standard error $=0.258)$ and after two sessions (lag $=-2$ ) the language characteristic distractive (cross-correlation coefficient $=-0.483$; standard error $=$ 0.243)

Question (e) examines possible patterns between the EEG variables and the language characteristics. For this purpose, canonical correlations were calculated [21-24]. The canonical correlation combines the multiple regression and factor analysis. First, the variables are assigned to so-called sets. A set encompasses the hypothesisled classification of variables. Variables, in which inconsistencies or distracters are substantiated, were inserted in Set 1. These variables are: Mean Theta (VAR1, respectively VAR00001), Mean Alpha (VAR2 respectively VAR00002), Mean Beta (VAR4 respectively VAR00004) and distractive. Those variables, which cause coherences were inserted in the $2^{\text {nd }}$ set. These variables are: Mean- SMR (VAR3 respectively VAR00003), mean-EMG (VAR5 respectively VAR00005), supportive and central.

Following a weighted linear combination between the variables of both sets will be drawn up in such manner, that their correlation will become maximal. Its weights are called canonical coefficients. A further linear combination from the residuals is created and correlated. The values of the variables from one set, estimated by regression analysis, can be correlated with the actual measurements of these variables. Their correlation coefficients are the canonical factor scores of the variables of the respective linear combination of the respective sets.

The results for the question (b) and (c) indicate that both subjects have used different strategies during therapy:
Subject B.Z. used a process-oriented strategy for her changes in condition. The learning mechanism of B.Z. is called habituation. This means that B.Z. used a progressive recall of past experiences in order to get coherence. B.Z. integrates past experiences into a single factor. This factor basically represents a "priority list" from past experiences. In the process-oriented strategy, a single significant canonical correlation is to be expected.

Subject K.T. used a structure-oriented approach for the change of condition. T.K.'s learning mechanism is an adaptive. K.T. has learned to adjust every time to the heterogeneous situational requirements. K.T. does not have a "priority list" from past experiences. The structureoriented strategy of K.T. is based on multiple current comparisons with all the variables. In the canonical correlation analysis several significant canonical correlations are to be expected.

\section{Results for B.Z.}

\begin{tabular}{|c|c|}
\hline Canonical & Correlations \\
\hline 1 & 0.993 \\
\hline 2 & 0.733 \\
\hline 3 & 0.482 \\
\hline 4 & 0.050 \\
\hline
\end{tabular}

Test that remaining correlations are zero:

\begin{tabular}{|c|c|c|c|c|}
\hline & Wilk's & Chi-SQ & DF & Sig. \\
\hline 1 & 0.005 & 76,359 & 16,000 & 0.000 \\
\hline 2 & 0.354 & 15,040 & 9,000 & 0.090 \\
\hline 3 & 0.766 & 3,862 & 4,000 & 0.425 \\
\hline 4 & 0.997 &, 037 & 1,000 & 0.848 \\
\hline
\end{tabular}

The Wilk's $\lambda$ - test conveys, that only the first canonical correlation $(=0.993)$ is significant.

\section{Standardized Canonical Coefficients for Set-1}

\begin{tabular}{|c|c|c|c|c|}
\hline & $\mathbf{1}$ & $\mathbf{2}$ & $\mathbf{3}$ & $\mathbf{4}$ \\
\hline VAR00001 &, 022 &,- 473 &, 791 &,- 653 \\
\hline VAR00002 &,- 031 &,- 929 &,- 649 &, 046 \\
\hline VAR00004 &, 019 &, 806 &,- 695 &,- 563 \\
\hline distrB &, 991 &,- 074 &,- 560 &,- 043 \\
\hline
\end{tabular}

Standardized Canonical Coefficients for Set-2

\begin{tabular}{|c|c|c|c|c|}
\hline & $\mathbf{1}$ & $\mathbf{2}$ & $\mathbf{3}$ & $\mathbf{4}$ \\
\hline VAR00003 &, 048 &, 055 &, 098 & $-1,152$ \\
\hline VAR00005 &, 071 &, 810 &,- 681 &, 452 \\
\hline centB &,- 897 &,- 140 &,- 873 &, 097 \\
\hline supB & $-1,101$ &, 588 &, 015 &,- 205 \\
\hline
\end{tabular}

The factor loadings for set 1 and set 2 are as follows:

\section{Canonical Loadings for Set-1}

\begin{tabular}{|c|c|c|c|c|}
\hline & $\mathbf{1}$ & $\mathbf{2}$ & $\mathbf{3}$ & $\mathbf{4}$ \\
\hline VAR00001 &, 144 &,- 300 &, 362 &,- 871 \\
\hline VAR00002 &,- 392 &,- 666 &,- 587 &,- 242 \\
\hline VAR00004 &,- 275 &, 296 &,- 466 &,- 787 \\
\hline distrB &, 999 &,- 020 &,- 017 &, 027 \\
\hline
\end{tabular}

Canonical Loadings for Set-2

\begin{tabular}{|c|c|c|c|c|}
\hline & $\mathbf{1}$ & $\mathbf{2}$ & $\mathbf{3}$ & $\mathbf{4}$ \\
\hline VAR00003 &, 243 &, 157 &,- 373 &,- 882 \\
\hline VAR00005 &, 394 &, 755 &,- 525 &, 008 \\
\hline centB &,- 291 &,- 561 &,- 768 &,- 104 \\
\hline supB &,- 635 &, 513 &, 575 &, 047 \\
\hline
\end{tabular}


The first factor set 1 includes positive charges for the language variable "distractive" and for a reduced alertness (mean Theta = Var00001). It contains negative charges for Hypervigilance (Mean Beta $=$ Var00004) and for the inhibitory mechanism of attention (Mean Alpha $=$ Var00002). The lesser the vigilance, the higher the relaxation and sleep disposition and the higher the probability for irrelevant details. This factor could be seen as a factor of instability and irritability.

The first factor of set 2 is characterized by negative charges of the language characteristics supportive and central and by positive charges of the EEG characteristics Mean EMG (= Var00005) and SMR (= Var00003). The higher the physical balance, the lower the probability to focus on relevant details. This factor could be described as relaxed, attentive balance.

The fact, that this charges result out of a requirement to achieve a maximal correlation between both sets needs to be respected. These charges are the correlations between the estimated and actual measured values. This canonical correlation coefficient between both sets means that irritability on the one hand is compensated by a balance on the other. This explains the negative charges for "central" and "supportive". The compensation of the first factors of set 1 ; hence that of irritability requires a resource-based physiological balance. This resource-oriented balance represents factor 1 of set 2 .

Another argument in favor of this hypothesis is: irritability (factor 1, set 1) means that the focus of attention is on distractors. Compensation by a resource-oriented physiological balance (factor 1, set 2) represents a significant correlation between the factor scores of factor 1 set 2 . The variables of factor 1 , set 1 do not correlate significantly with each other or with the factor scores of the first factor set 2 .

$(\mathrm{r}$ (Factor scores/mean theta $)=-0.025 ; \mathrm{r}$ (Factor scores $/$ mean alpha $=0.197 ; \mathrm{r}$ (factor scores $/$ mean beta $)=0.053)$. The correlation of the variable "distractive" and the factor scores of the first factor set 2 equals $\mathrm{r}=0.369(\mathrm{p}=.109)$. If on the other hand, the variables Mean Theta, Mean Alpha and beta are taken out of variable "distractive", a partial correlation of $\mathrm{r}=0.527(\mathrm{p}=0.030)$ and in a regression analysis a $\mathrm{R}=$ $0.911(\mathrm{~F}=41,22 ; \mathrm{DF}=2, \mathrm{p}=0.000919$ and $\mathrm{a} \beta$ - coefficient $\beta=0.369$ $(\mathrm{T}=3.683 ; \mathrm{p}=0.002)$ can be seen. This result means: a resource-based physiological balance compensates for the irritability in the sense of a distraction from attention [25].

Results of subject K.T.

\begin{tabular}{|c|c|}
\hline Canonical & Correlations \\
\hline 1 &, 997 \\
\hline 2 &, 987 \\
\hline 3 &, 781 \\
\hline 4 &, 478 \\
\hline
\end{tabular}

Test that remaining correlations are zero:

\begin{tabular}{|c|c|c|c|c|}
\hline & Wilk's & Chi-SQ & DF & Sig. \\
\hline 1 &, 000 & 146,255 & 16,000 &, 000 \\
\hline 2 &, 008 & 70,849 & 9,000 &, 000 \\
\hline 3 &, 301 & 17,412 & 4,000 &, 002 \\
\hline 4 &, 771 & 3,766 & 1,000 &, 052 \\
\hline
\end{tabular}

The Wilk's $\lambda$ - test conveys that the first three canonical correlations are significant. This prompts the following weights for linear combination:

\section{Standardized Canonical Coefficients for Set-1}

\begin{tabular}{|c|c|c|c|c|}
\hline & $\mathbf{1}$ & $\mathbf{2}$ & $\mathbf{3}$ & $\mathbf{4}$ \\
\hline VAR1KT &,- 303 &,- 203 &,- 452 & 6,326 \\
\hline VAR2KT &, 431 & 1,135 & 2,397 & $-6,286$ \\
\hline VAR4KT &,- 047 &, 069 & $-2,179$ &, 116 \\
\hline distrT & $-1,006$ &, 147 &,- 126 &, 252 \\
\hline
\end{tabular}

Standardized Canonical Coefficients for Set-2

\begin{tabular}{|c|c|c|c|c|}
\hline & $\mathbf{1}$ & $\mathbf{2}$ & $\mathbf{3}$ & $\mathbf{4}$ \\
\hline VAR3KT &, 254 & 1,309 & 3,028 & $-1,185$ \\
\hline VAR5KT &,- 164 &,- 351 & $-3,347$ &, 813 \\
\hline centT & 1,374 &,- 201 &, 354 &, 696 \\
\hline supT & 1,473 &,- 263 &, 309 &,- 455 \\
\hline
\end{tabular}

Factors loadings for set 1 and set 2 are as follows:

\section{Canonical Loadings for Set-1}

\begin{tabular}{|c|c|c|c|c|}
\hline & $\mathbf{1}$ & $\mathbf{2}$ & $\mathbf{3}$ & $\mathbf{4}$ \\
\hline VAR1KT &, 186 &, 961 &, 037 &, 200 \\
\hline VAR2KT &, 151 &, 987 &, 030 &, 041 \\
\hline VAR4KT &, 185 &, 881 &,- 435 &, 006 \\
\hline distrT &,- 995 &, 092 &, 022 &,- 043 \\
\hline
\end{tabular}

\section{Canonical Loadings for Set-2}

\begin{tabular}{|c|c|c|c|c|}
\hline & $\mathbf{1}$ & $\mathbf{2}$ & $\mathbf{3}$ & $\mathbf{4}$ \\
\hline VAR3KT &, 189 &, 974 &,- 119 &,- 032 \\
\hline VAR5KT &, 239 &, 882 &,- 406 &,- 011 \\
\hline centT &, 300 &, 362 &, 158 &, 869 \\
\hline supT &, 393 &,- 407 &,- 172 &,- 806 \\
\hline
\end{tabular}

Factor 1 of set 1 represents the language characteristic distractive; due to its negative charge, it could be labeled as a factor of linguistic regulation. Factor 2 contains the EEG variables Mean Alpha, Mean Theta and Mean Beta. It could be labeled as a factor of physiological imbalance. The third factor could, due to its high negative charge of Mean Beta, be labeled as a down regulation of attention.

Factor 1 of set 2 contains productive language characteristics. It could be described as a factor of linguistic consistency. Factor 2 represents the corresponding EEG features and language characteristics. It could be described as a factor of the psycho-physiological balance. The language characteristic "supportive" has an "intermediate role". It impacts factor 1 positively and factor 2 negatively. It is a characteristic of the support of the central category. Factor 3 could be described as a factor of autonomous coherence; the physiological characteristics of the relaxed attention show a negative charge.

The balance is based on the correlations of the three factors between the two sets. Regulation of irrelevant linguistic details correlates with the factor of the central linguistic characteristics. Psycho-physiological imbalance correlates with psychological balance and coherence. Factor 3 , the autonomous coherence, correlates with a factor of reduced hyper-vigilance.

\section{Conclusion/ Discussion}

This study was able to confirm that the general functional level as well as the ability to pay attention can be improved in subjects displaying the negative symptoms of schizophrenia by using neurofeedback training.

The study shows differential strategies of change of the concerned subjects. Person K.T. uses a learning mechanism that could be described 
as the adaptation; person B.Z. uses a learning mechanism that could be described as a habituation. A change of status through adaptation is the adjustment of the current, real state unto the anticipated status. The habituation is the adaptation of the current, tangible status to the retrospective regularity of previous changes in status. The subjects use different reference systems:

Subject K. T. orients himself, different from B.Z., in every session on currently present patterns of the individual EEG - Variables. The difference can be seen in the significant differences between the product-moment - correlations between both subjects.

Subject B. Z. orients himself on the standard of the EEG - variables in the previous therapeutic sessions. This difference is reflected in the different cross-correlations between the subjects.

In the language protocols of B.Z. the product-moment- correlations show negative correlations in between central and supportive between supportive and distractive. These correlations correspond to the assumptions made regarding the coherence. In regard to K.T. the product-moment-correlations show a negative relation between central and supportive and the cross-correlations show a negative relation between central and supportive and between central and distractive. These results correspond to the assumptions made regarding the coherence as well.

The coherence assumption is $\mathrm{b}$ nevertheless contradicted by one positive correlation $(r=0.658 ; p=0.002)$ between the duration of treatment and the category distractive in the case of B.Z. and a negative correlation between duration of therapy and central $(\mathrm{r}=-0.476 ; \mathrm{p}=$ 0.034 ) in the case of K. T. Concerning K.T., the categories of supportive and distractive do not correlate significantly with the duration of therapy. In the case of B.Z., the category supportive show a significant negative correlation with $(r=-0.459 ; \mathrm{p}=0.042)$ with the duration of therapy.

This finding could be explained through a balance - hypothesis. The decrease in central important characteristics and the increase in the number of extraneous details could be based on a continuous oscillation of relevant and irrelevant clues. This "balance" is the result of a further finding of this study. B.Z. strives for a "cortical" balance between EEG characteristics and language characteristics. This cortical balance is reflected in the cross-correlations between the EEG variables and the voice characteristics. Mean Beta increases the frequency of the category central (lag -3) and reduces the frequency of category central (lag-2). Central, in return, reduces Mean Beta retroactively. Increased Mean Beta reduce the frequency of the category supportive (lag-3) and increase the category supportive (lag-2). Mean Alpha reduces the likelihood of the category supportive whilst simultaneously increasing the category distractive (lag-6). Balances are

- Oscillations between alternatives and/or different directional impacts

- that are simultaneous or offset or they are

- feedback regulations.

"The brain generates its own temporal structure, which is largely organized by oscillations" [6].

In the canonical correlation analyzes of both subjects, heterogeneous psycho-physiological combinations of characteristics are correlated. The analysis shows that K.T.'s processes are more complex than those of B.Z. The assumption is that K.T. compensates labile characteristics with stable characteristics. Simultaneous comparison processes lead to unstable balances.

Regarding K.T., the foundation of coherence results in a psychophysiological balance. This balance is based on the simultaneous integration and correlation of heterogeneous feature configurations. Concerning B.Z foundation of coherence results in a psychophysiological balance. This balance is based on the gradual regulation of differential characteristic sequences.

\section{References}

1. Omanson RC (1982) An Analysis of Narratives: Identifying Central, Supportive and Distracting Content. Discourse Processes 5: 195-224.

2. Pazooki K, Leibetseder M, Renner W, Gougleris G, Kapsali E (2019) (zur Veröffentlichung eingereicht). Neurofeedback Treatment of Negative Symptoms in Schizophrenia. A Case Report. Appl Psychophysiol Biofeedback 44: 31-39. [Crossref]

3. Andreasen NC (1986) Scale for the Assessment of Thought, Language, and Communication (TLC). Schizophr Bull1 2: 473-482. [Crossref]

4. Andreasen NC, Grove WM (1986) Thought, Language, and Communication in Schizophrenia: Diagnosis and Prognosis. Schizophr Bull 12: 348-359. [Crossref]

5. Hoffman RE, Stopek S, Andreasen NC (1986) A comparative study of manic vs schizophrenic speech disorganization. Arch Gen Psychiatry 43: 831-838. [Crossref]

6. Klimesch W (2012) Alpha-band oscillations, attention, and controlled access to stored information. Trends Cogn Sci 16: 606-617. [Crossref]

7. Hyun J, Baik MJ, Kang UG (2011) Effects of Psychotropic Drugs on Quantitative EEG among Patients with Schizophrenia-spectrum Disorders. Clin Psychopharmacol Neurosci 9: 78-85. [Crossref]

8. Heinrich H, Gevensleben H, Strehl U (2007) Annotation: Neurofeedback - train your brain to train behaviour. J Child Psychol Psych 48: 3-16. [Crossref]

9. Döring N, Bortz J (2016) Datenerhebung. In: N. Döring \& J. Bortz: Forschungsmethoden und Evaluation in den Sozial- und Humanwissenschaften. Berlin: Springer Verlag 321577 .

10. Söderberg P, Tungström S, Armelius BÅ (2005) Reliability of Global Assessment of Functioning Ratings Made by Clinical Psychiatric Staff. Psychiatr Serv 56: 434-438. [Crossref]

11. Schäfer S (2010) Immer öfter eine gute Wahl. Lizenzfreie Testverfahren. Psychotherapie Aktuell 3: 12-16.

12. Startup M, Jackson MC, Bendix S (2002) The concurrent validity of the Globa Assessment of Functioning (GAF). Br J Clin Psychol 41: 417-422. [Crossref]

13. DSM-IV (1996) Diagnostisches und Statistisches Manual Psychischer Störungen Deutsche Bearbeitung und Einführung von $\mathrm{H}$. Saß, H-U- Wittchen und m. Zaudig. Göttingen: Hogrefe.

14. Lange C, und Heuft G (2005) GAF - Skala zur globalen Erfassung des Funktionsniveaus. In: B. Strauß und J. Schumacher, (Hrsg). Klinische Interviews und Ratingskalen. Göttingen: Hogrefe pp.143-148.

15. Woldoff SB (2004) Reliability of Global Assessment of Functioning Scale. Unpublished Doctoral Disseration: Philadelphia: Drexel University.

16. Deschner MS (2010) Entwicklung und Evaluation einer kompensatorisch-kognitivbehavioralen Einzeltherapie für ADHS im Erwachsenenalter. Dissertation, Universität Mannheim

17. Prieler J (2011) Computerized Attention and Concentration Tests - Simple Reaction CompACT-526 SR. Göttingen (Germany): Hogrefe.

18. Bühner M (2011) Einführung in die Test- und Fragebogenkonstruktion. München Pearson.

19. Huber HP (1973) Psychometrische Einzelfalldiagnostik [psychometric single case 489 diagnostics]. Weinheim, Basel (Switzerland): Beltz.

20. Bortz J (1977) Lehrbuch der Statistik. Für Sozialwissenschaftler [Textbook of statistics for social scientists]. Berlin (Germany): Springer. 
21. Bortz J, und Schuster Ch (2010) Statistik für Human- und Sozialwissenschaftler, Berlin: Springer.

22. Eckey H-F, Kosfeld R, und Rengers (2002) Multivariate Statistik. Wiesbaden: Verlag Dr. Th. Bader GmBH.
23. Schendera Ch, (2014) Regressions-Analyse mit SPSS. München: Oldenburg Wissenschaftsverlag.

24. Wentura D, Pospeschill M (2015) Multivariate Datenanalyse. Wiesbaden Springer.

25. Eid M, Gollwitzer M, Schmitt M (2011) Statistik und Forschungsmethoden. Weinheim: Beltz Verlag.

Copyright: $\mathbb{C} 2019$ Leibetseder M. This is an open-access article distributed under the terms of the Creative Commons Attribution License, which permits unrestricted use, distribution, and reproduction in any medium, provided the original author and source are credited. 